\title{
STIMULATED RAMAN OSCILLATION IN CAPILLARY WAVEGUIDE RESONATORS
}

\author{
A.J. BERRY' and D.C. HANNA \\ Department of Physics. University of Southamptom, Highficld, Southampton, SO9 SNH, UK
}

Recejved 26 January 1983

\begin{abstract}
The threshold for stimulated Raman scattering in $\mathrm{H}_{2}$ gas using a $1.06 \mu \mathrm{m}$ pump has been reduced by a factor of 30 (to $\sim 40 \mathrm{~kW}$ in a $35 \mathrm{~ns}$ pulse) by the combined use of a capillary waveguide and a resonator providing Stokes feedback. Similar enhancement has been observed in $\mathrm{Cl}_{4}$ where, in addition, the use of a dispersive resonator has allowed suppression of stimulated Brillouin seattering even with single mode pumping.
\end{abstract}

The application of capillary waveguide techniques [1] to stimulated Raman scattering (SRS) was first reported by Rabinowitz et al. [2] and Hartig and Schmidt [3]. In a recent paper [4] we gave results of an investigation into the threshold reduction that can be achieved by the use of such waveguides. In this paper we report results obtained with the combined use of waveguiding and feedback of the Stokes wave. With this waveguide resonator the threshold for SRS in $\mathrm{H}_{2}$ gas was reduced to $\sim 40 \mathrm{~kW}$ for a $1.06 \mu \mathrm{m}$ pump (Stokes wavelength $1.9 \mu \mathrm{m}$ ) corresponding to a reduction of threshold by a factor of 30 compared with the case of an unguided gaussian beam pump without Stokes feedback. A simple analytical model of the Raman oscillator gives good agreement with the experimental results and thus suggests that the analysis should provide a useful guide in extrapolating to different experimental conditions (e.g. different pump wavelength, guide dimension, resonator length. pump pulse duration etc.) The low threshold is atractive since it brings the technique of SRS within reach of lasers of modest power. An interesting example would be the use of a $\mathrm{Cu}$ vapour pumped dye laser as the pump for a waveguide . Raman laser, offering a pulsed source of high repetition rate and wide infrared tunability via multiple Stokes shifts [3]. Besides leading to a reduction of threshold the use of an oscillator configuration can provide further advantages. For example, we have used a dispersive Raman resonator, containing a prism, to suppress Brillouin feedback when using $\mathrm{CH}_{4}$ as the Raman medium. With this technique, $\mathrm{CH}_{4}$ now looks a more attractive Raman medium since it has suffered from the twin disadvantages of a high threshold for Raman scattering and a relatively low threshold for Brillouin scattering.

The pump laser used in these experiments was a $\mathrm{TEM}_{00}$ mode Q-switched NdYAG laser operating in a single longitudinal mode, as described in [5], giving an output energy up to $100 \mathrm{~mJ}$ in a pulse of duration $\tau$, between 30 and $40 \mathrm{~ns}$. Various capillary dimensions have been tried but typically had a length $l \sim 30 \mathrm{~cm}$, and an inside diameter $(2 a)$ of $\sim 200 \mu \mathrm{m}$. Usually the capillary was in the form of a thin-walled tube $(\sim 300$ $\mu \mathrm{m}$ outside diameter) inserted into a supporting capillary of inside diameter $\sim 300 \mu \mathrm{m}$ and outside diameter $\sim 5 \mathrm{~mm}$ [4]. This was then placed inside a pressure vessel consisting of a steel tube equipped with end fittings holding Brewster angled fused silica windows to suppress window feedback. The Brewster windows were spaced by $44 \mathrm{~cm}$, thus leaving a gap of $\sim 7 \mathrm{~cm}$ between capillary and window at each end. In all our measurements the pump was focussed into the capillary to satisfy the condition $3 w_{0}=2 a[6]$. A representative measurement of Raman threshold in $\mathrm{H}_{2}$ for a single pass through the capillary (i.e. without feedback) was as follows: $l=27.5 \mathrm{~cm}, 2 a=200 \mu \mathrm{m}$, pump transmission $T=60 \%$, gas pressure $25 \mathrm{~atm}$, threshold energy $9.1 \mathrm{~mJ}$, hence peak power $260 \mathrm{~kW}$ $(\tau=33 \mathrm{~ns})$. With some simplifying assumptions a 
theoretical prediction of threshold can be made which agrees well with the experimental results. We assume that the Stokes loss and gain within the capillary can be separated so that the net Stokes gain is

$\exp \left(-2 \gamma_{\mathrm{s}} l\right) \exp \left\{\int_{0}^{l} \frac{2 P_{\mathrm{p}}(z)}{\pi W_{0}^{2}} f g d z\right\}$.

The Stokes field attenuation constant $\gamma_{s}$ is assumed to be given by the theoretical value from Marcatili and Schmeltzer [1] whereas the pump field attenuation constant $\gamma_{\mathrm{p}}$, where

$P_{\mathrm{p}}(z)=P_{\mathrm{p}}(0) \exp \left(-2 \gamma_{\mathrm{p}} z\right)$

is derived from the measured pump transmission. (This experimentally derived value of $\gamma_{p}$ is usually significantly greater than the theoretical Marcatili and Schmeltzer value, most probably due to imperfect capillary straightness). The factor $g$ is the usual plane wave Raman gain coefficient (see e.g. Trutna and Byer [7]). The quantity $2 P_{\mathrm{p}}(z) / \pi w_{0}^{2}$ is the on-axis pump intensity and the numerical factor $f$ accounts for the transverse variation of pump and Stokes fields via an overlap integral as discussed by Boyd et al. [8]. Taking the pump and Stokes waves to be both pure $\mathrm{EH}_{11}$ modes of the guide gives $f=0.56$ [9], (if they are both assumed to be gaussian beams of the same spotsize one obtains $f=0.5[8]$ ), and by equating the net Stokes gain at threshold to $\exp (30)$, it is found from (1) and (2) that the threshold pump power $\boldsymbol{P}_{\mathrm{p}, \mathrm{th}}$ is

$P_{\mathrm{p}, \mathrm{th}}=\pi W_{0}^{2}\left(30+2 \gamma_{\mathrm{s}} l\right) / 1.12 g l_{\mathrm{eff}}$,

where

$l_{\mathrm{eff}}=\left[1-\exp \left(-2 \gamma_{\mathrm{p}} l\right)\right] / 2 \gamma_{\mathrm{p}}$.

Using (3) and (4) for the experimental conditions re. ferred to above, a predicted threshold power of 230 $\mathrm{kW}$ is obtained.

In the case of a resonator configuration we have again used this same analysis to calculate the net gain for each transit through the capillary.

Various resonator configurations have been used and fig. 1 shows one arrangement. The pump beam passes through a plane resonator mirror having a transmission of $\sim 90 \%$ at $1.06 \mu \mathrm{m}$ and total reflection at the Stokes wavelength $(1.907 \mu \mathrm{m})$. The second resonator mirror also transmitted most of the pump and

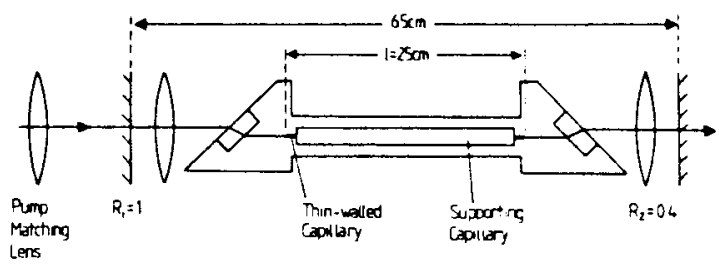

Fig. 1. Capillary waveguide resonator.

had a reflectivity of $40 \%$ at the Stokes wavelength. Intra cavity lenses of focal length $15 \mathrm{~cm}$ were placed close to the mirrors to match the resonator to the as. sumed Stokes spotsize leaving the capillary (i.e. $3 W_{0}=2 a$, as for the pump). With this arrangement and a pump pulse of $36 \mathrm{~ns}$ FWHM, threshold was reached at $40 \mathrm{~kW}$ pump power into the capillary. This represents a factor of $\sim 6$ reduction in threshold as a result of the Stokes feedback and a factor of 30 re. duction compared with the case for an unguided pump without Stokes feedback. We have also tested a much simpler resonator configuration in which the feedback was provided by two uncoated plane reflec. tors very close (i.e. within a few $\mathrm{mm}$ ) to the ends of the capillary. This was achieved by using plane glass flats as the windows of the high pressure cell, and ensuring that the capillary filled the space between them.

This arrangement gave a threshold of $\sim 90 \mathrm{~kW}$ for a capillary of internal diameter $330 \mu \mathrm{m}$, and $42 \mathrm{~kW}$ for a capillary of $200 \mu \mathrm{m}$ diameter. When pumped at twice the threshold level, the $200 \mu \mathrm{m}$ capillary gave a measured photon conversion efficiency to first Stokes of $15 \%$ (9\% in the forward direction. $6 \%$ in the backward direction). On the other hand. observation of the transmitted pump intensity showed almost total depletion of the pump over most of the pulse and it is not yet clear why the measured conversion efficiency was not greater. The calculated threshold powers for the oscillat or were in good agree. ment with a simple model. It was assumed in this model that Stokes feedback was determined solely by the mirror reflections $R_{1}, R_{2}$, that the maximum Stokes gain from multiple resonator transits would be experienced by those Stokes photons travelling forward at the peak of the pump pulse, and that the pump pulse power has a gaussian time dependence $P(t)=\exp \left[-\left(t / \tau^{\prime}\right)^{2}\right]$ where $\tau^{\prime}=\tau_{\text {FWHIM }} / 2 \sqrt{1 \mathrm{n} 2}$. With these assumptions, the condition that threshold is reached with $n$ resonator transits, with a pulse peak 
power $P_{\text {th }}$. becomes

$P_{\mathrm{th}}=\left\{\pi \mathrm{H}_{0}^{\prime 2}\left[30+2 m \gamma_{\mathrm{s}} l-[(n-1) / 2] \ln \left(R_{1} R_{2}\right)\right]\right\}$

$X\left\{1.12 l_{\mathrm{ef}}\left[g_{\mathrm{f}}+g_{\mathrm{B}} \exp \left[-\left(T / \tau^{\prime}\right)^{2}\right]+g_{\mathrm{B}} \exp \left[-\left(T / \tau^{\prime}\right)^{2}\right]\right.\right.$

$\left.\left.+g_{\mathrm{F}} \exp \left[-\left(2 T / \tau^{\prime}\right)^{2}\right]+g_{\mathrm{F}} \exp \left[-\left(2 T / \tau^{\prime}\right)^{2}\right]+\ldots\right]\right\}^{-1}$,

where $g_{F} \cdot g_{B}$ are respectively the forward and backward Raman gain coefficients, $2 T$ is the time for a resonator round trip, and the sum in the denominator continues to $n$ tems. One can now vary $n$ to obtain the minimum $P_{\text {th }}$ from (5) and this then represents the predicted oscillat or threshold. For the resonator of fig. 1, the predicted threshold is $\sim 30 \mathrm{~kW}$ whereas the observed threshold was $\sim 40 \mathrm{~kW}$. For the plane/plane resonator it was not obvious what values of reflectivity to use for the window's, as one of these had parallel faces and the other had a $5 \mathrm{mrad}$ wedge. Also. they were only approximately normal to the capillary axis. Fig. 2 shows the observed threshold as a function of gas pressure and the predicted thresholds for assumed reflectivities of $4 \%$ and $4 \%$ at the two end windows. Again the agreement is good, with the model coping adequately with changes in capillary bore diameter. It should be noted, however, that the predicted threshold is fairly insensitive to the assumed values of Stokes feedback.

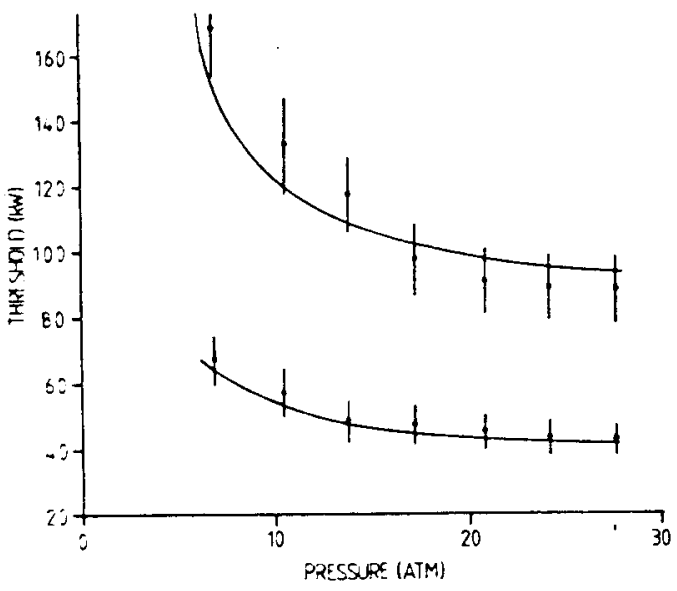

Fig. 2. $1.06 \mu \mathrm{m}$ pump threshold power (kW) versus $\mathrm{H}_{2}$ gas pressure (atmospheres) for resonator with plane uncoated reflectors butted against the waveguide ends. Upper curve is for $330 \mu \mathrm{m}$ bore capillary, lower curve is for $200 \mu \mathrm{m}$ bore. Solid lines are calculated using eq. (5).
As a further test of the waveguide/resomator we investigated stimulated Raman scattering in $\mathrm{CH}_{4}$ gas. The case of $\mathrm{CH}_{4}$ as a Raman medium presents some problems for pump wavelengths in the near infrared since the threshold is higher than for $\mathrm{H}_{2}$ (the peak Raman cross-section is about a factor of 4 less than for $\mathrm{H}_{2}$ at $\sim 12$ atm [10] and a factor of $\sim 2$ less at $\sim 30 \mathrm{~atm})$. In addition the stimulated Brillouin scattering threshold is rather low, leading to competition between SRS and SBS [11]. Murray et al. [12] show that, assuming the Raman linewidth to be independent of pressure, the ratio of Brillouin gain to Raman gain for a Raman Stokes frequency $\nu_{\mathrm{s}}$ is given by $g_{\mathrm{SBS}} / g_{\mathrm{R}}=C \rho / \nu_{\mathrm{s}}$ where $\rho$ is the density in amagats and $C$ is a factor depending on the particular gas molecule involved. This factor $C$ can be determined from the results of Minck et al. [11] for the case of a ruby laser pump and $\mathrm{CH}_{4}$. One can then predict that for a $1.06 \mu \mathrm{m}$ pump the ratio is $g_{\mathrm{SBS}} / g_{\mathrm{R}, \mathrm{F}}=\rho / 8.5, \rho$ again in amagats. Thus, a low enough pressure, say a few atmospheres, would discriminate against stimulated Brillouins scattering but would also imply a very high Raman threshold. Another way to discriminate against Brillouin scattering is to use a short pump pulse or a multimode pump pulse which contains temporal structure of short duration. The forward Raman Stokes wave can take advantage of the high intensity in one of the pump pulse spikes whereas the backward travelling Brillouin Stokes wave only experiences the average pump intensity. Thus, by using high pressures (30-60 atm) and a multimode Nd: YAG pump, Bruns et al. [13] have achieved efficient conversion to the first Stokes wave at 1.544 $\mu \mathrm{m}$. The waveguide/resonator scheme allows another approach. Since a low Raman threshold can be achieved it is possible to work with a low pressure and also add extra discrimination against the Brillouin Stokes wave by means of frequency selection in the resonator. The resonator configuration we have used is the same as in fig. 1 but with a $60^{\circ}$ prism placed between the lens and the output mirror $(R=0.5$ at $1.544 \mu \mathrm{m})$. The output mirror was aligned to provide feedback of the Raman Stokes wave thus suppressing Brillouin Stokes feedback. With a $\mathrm{CH}_{4}$ pressure $\sim 6$ atm the measured Raman threshold power (and this is using a single longitudinal mode pump pulse) was $180 \mathrm{~kW}(6.2 \mathrm{~mJ}$ in $32 \mathrm{~ns}$ FWHM), whereas the calculated threshold using the previous analysis was 200 
$k W$. No Brillouin scattering was observed until pump powers in excess of $500 \mathrm{~kW}$ were reached. At a pump intensity of $2.5 \times$ threshold the 1st Stokes output was measured to be $\sim 330 \mu \mathrm{J}$. No sign of thermal blooming was observed even at pulse repetition rates of $20 \mathrm{~Hz}$, whereas in the scheme reported by Bruns et al. this was evident and necessitated gas circulation. A further advantage of the waveguide/resonator approach is that it provides a diffraction limited output whereas the output from the Raman laser of Bruns et al. was multimode. The threshold requirement for our $\mathrm{CH}_{4}$ Raman laser is small enough to leave a very substantial amount of pump energy for pumping a Raman amplifier cell. This arrangement offers advantages for efficient Raman conversion [14] and in particular it means that the amplifier can be pumped at a level below that required for SBS. We anticipate that efficient conversion from $1.06 \mu \mathrm{m}$ to $1.544 \mu \mathrm{m}$ could then be achieved with a diffraction limited Stokes output.

In conclusion, we have demonstrated low threshold operation of a Raman laser by the combined use of a capillary waveguide and a resonator to provide Stokes feedback. An obvious benefit of the reduced threshold is that efficient stimulated Raman scattering is now within reach of pump lasers of much lower power. One can also consider further exploration of the waveguide resonator concept, for example using a ring resonator in which the Raman medium is synchronously pumped by a mode locked pulse train [15]. $\mathrm{CH}_{4}$ gas should be well suited to this scheme as it has a short $T_{2}$ and a large integrat. ed Raman cross section. We plan to perform experiments to evaluate this scheme. In fact it would seem to us that there is now a case for re-examining a number of nonlinear processes in gases to see whether they may find wider application as a result of the en- hancement that waveguide/resonator techniques can offer.

This work has been supported by the Science and Engineering Research Council and one of us (A.J.B.) holds a CASE studentship sponsored by J.K. Lasers Ltd. We acknowledge valuable discussions with M.C. Ibison.

\section{References}

[1] E.A.J. Marcatili and R.A. Schmeltzer, Bell Syst. Tech:J. 43 (1964) 1783.

[2] P. Rabinowitz, A. Kaldar, R. Brickman and W. Schmidt, Appl. Optics 15 (1976) 2006.

[3] W. Hartig and W. Schmidt, Appl. Phy's. 18 (1979) 235.

[4] A.J. Berry, D.C. Hanna and D.B. Hearn, Optics Comm. 43 (1982) 229.

[5] A.J. Berry, D.C. Hanna and C.G. Sawyers, Optics Comm. 40 (1981) 54.

[6] R.L. Abrams, IEEE J. Quant. Electron. QE-8 (1982) 838.

[7] W.R. Trutma and R.L. Byer, Appl. Optics 19 (1980) 301.

[8] G.D. Boyd, W.D. Johnson and I.P. Kaminow, IEEE J. Quantum Electron QE-5 (1969) 203.

[9] M.C. Ibison (private communication).

[10] W.R. Fenner, H.A. Hyatt, K.M. Kellam and S.P.S. Porto, J. Opt. Soc. Am. 63 (1973) 73.

[11] R.W. Minck, E.E. Hagenlocker and W.G. Rado. J. Appl. Phys. 38 (1967) 2254.

[12] J.R. Murray, J. Goldhar, D. Eimerl and A. Szoke, IEEE J. Quantum Electron QE-15 (1979) 342.

[13] D.G. Bruns, H.W. Bruesselbach, H.D. Stoval and D.A. Rockwell, IEEE J. Quantum Electron QE-18 (1982) 1246.

[14] H. Komine, E.A. Stappaerts, S.J. Brosnan and J.B. West, Appl. Phys. Lett. 40 (1982) 551.

[15] M.J. Colles, Appl. Phy's. Lett. 19 (1971) 23. 